\title{
Determining the Positions of the Elements for the 3-2-1 Principle of Location in a Solidworks Add-in
}

\author{
Oleg Mihaylov \\ Faculty of Industrial Technology \\ Technical University of Sofia \\ Sofia, Bulgaria \\ inj.mihaylov@gmail.com
}

\begin{abstract}
This paper presents the methodology behind the positioning of modular fixtures (for the 3-2-1 principle in particular) in a CAFD application (add-in) for SolidWorks. The application is integrated into SolidWorks and it's used to automate the design process of modular fixturing devices. Part of it is a methodology for automated determination of the positions of the modular elements by using rule-based logic and mathematical formulas.
\end{abstract}

Keywords-add-in, computer-aided fixture design, fixtures, locating, solidworks

\section{INTRODUCTION}

A fixture is a work-holding or support device used in the manufacturing industry. Fixtures are used to securely locate (position in a specific location or orientation) and support the work, ensuring that all parts produced using the fixture will maintain conformity and interchangeability. Using a fixture improves the economy of production by allowing smooth operation and quick transition from part to part, reducing the requirement for skilled labor by simplifying how workpieces are mounted, and increasing conformity across a production run $[1,2]$. In addition to processing, they are also used in experiments at elevated regimes and increased duration of cutting, while retaining accuracy in the measurement [3].

Fixture selection and design takes a significant part of the total time necessary for technical and technological production preparation and the costs associated with fixture design and manufacture can account for $10 \%-20 \%$ of the total cost of a manufacturing system [4]. These costs relate not only to fixture manufacture, assembly, and operation, but also to their design. Hence there are significant benefits to be gained by reducing the design costs associated with fixturing.

Approximately $40 \%$ of rejected parts are due to dimensioning errors that are attributed to poor fixturing design [5]. Fixture design work is also tedious and timeconsuming. It often heavily relies on fixture design engineers' experience/knowledge and usually requires over 10 years manufacturing practice to design quality fixtures [6]. The design and manufacture of a fixture can take several days to complete when human experience and a try-and-error process is utilized in fixture design. By reducing the subjective influence of the human factor and the try-and-error process the design process can be improved significantly.

Modular fixturing systems are the most widely used flexible fixturing systems in manufacturing. They are based on standardization of their components and they are designed as groups of pre manufactured standard elements and units with relatively tight tolerances. Those elements can be assembled in variety of different fixtures for locating and clamping variety of different parts. Once all the needed processes are completed the fixtures can be disassembled and reassembled in other, completely different configurations [7]. This is the modular systems' biggest difference and biggest advantage over the dedicated fixtures as the latter are usually scraped after completing their task.

For years, applications of modular fixtures have led to significant technological and economical benefits including [8]:

1. Significant reduction of the lead time - applications of modular fixtures can reduce more than $80 \%$ of the production preparation time.

2. Reduction of the production cost - modular fixtures are reusable, thus replacing dedicated with modular fixtures leads to a significant reduction of the materials and manufacturing costs of fixtures, especially in job and batch productions.

3. The use of modular fixtures helps ensuring production quality - the standard modular components are manufactured with tight tolerances. Once they are assembled into a configuration, the positions of functional components can be locally adjusted thus providing a potential to improve the production quality.

4. The use of modular fixtures may extend the manufacturing equipment's capability and improve the production rate.

Modular fixtures are composed from baseplate on which are positioned different locating and clamping elements. All the system holds up a workpiece in a strict position during process operations. [9] Generally the design process for modular fixture devices consists of the correct selection of locating, supporting and clamping elements and their precise position on the base plate. The number and position of locating points must be such that a workpiece's six degrees of freedom (Fig.1a) are adequately constrained during machining [10] and there are a variety of conceptual locating point layouts that can facilitate this, such as the 3-2-1 locating principle, plane and pin-hole locating, long-pin locating, V-block locating (Fig.1b). 
a)
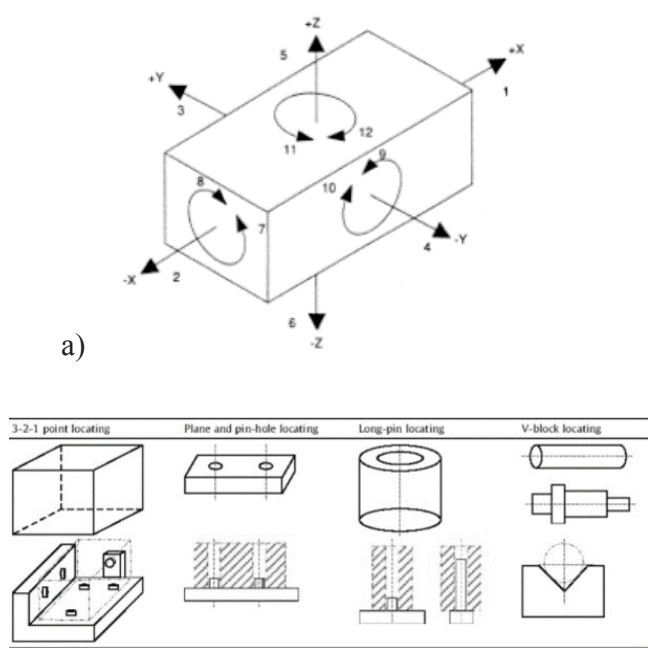

b)

Fig. 1. (a) The six degrees of freedom [11] and (b) methods of constraining them [12].

When separate locating elements are used (e.g. seating pins) their positions have to be such, that they ensure the stable position of the workpiece, i.e. the workpiece should not be moved by its own weight. This is achieved by placing the elements at maximum distance of one another [13]. When a baseplate with pin-hole pattern is used, there are obvious restrictions to that distance, so the positions of the locating elements have to be at maximum distance in contention with the pin-hole pattern and the workpiece's dimensions and form.

The automation of fixture design process is successfully solved by applying the system for Computer Aided Fixture Design (CAFD), which are using various intelligent methods, such as expert system, case based reasoning, and genetic algorithm (GA), etc.

The methodology presented in this paper uses rules of type IF-THEN-ELSE, mathematical formulas and SolidWorks' options to determine optimal positions for the locating elements.

\section{RELATED RESEARCHES.}

In [14] was presented the basis for an application integrated in SolidWorks, whose task is to create fixture design for locating prismatic and cylindrical parts by one of three methods - 3-2-1 locating principle, plane and pin-hole locating or V-block locating. The application is created like an integrated in SolidWorks' environment add-in and was developed by using SolidWorks' API, VB.NET programing language and rules IF-THEN-ELSE.

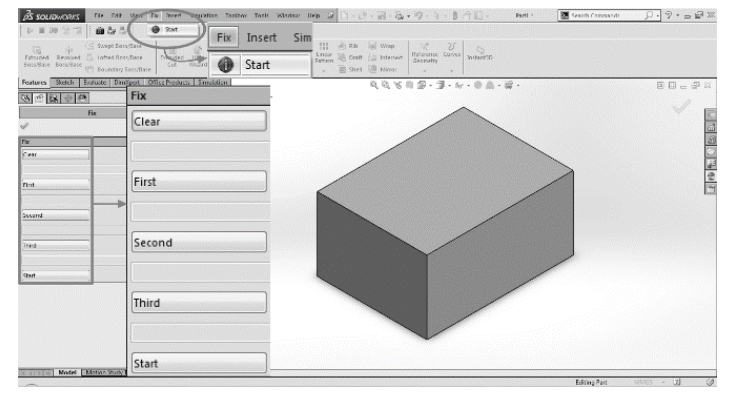

Fig. 2. Interface of the ADD-IN.

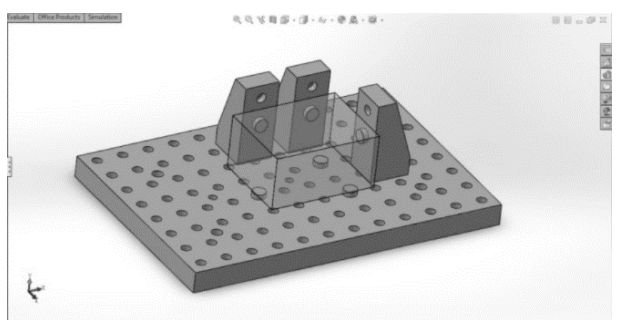

Fig. 3. Assembled fixture with workpiece.

The add-in is started in "PART" file by its own button in the Toolbar. The necessary input data is entered by the user by selecting the locating surfaces and confirming the selection with the buttons in the Property Manager Page (Fig.2.). While the selection is made, the program determines the surfaces' type and dimensions and the locating elements' positions. When the button "Start" is selected by the user the program creates a new assembly file, places the base plate and start inserting and mating operations for the rest of the elements and the part. After concluding all of its actions the program leaves the assembly file open for the user so he can continue working with it (Fig.3.).

\section{Selecting surfaces.}

To the aforementioned interface (Fig.3.) was added a button for the selection of the machined surface (Fig.4.). Whit this button the user marks the surface(s) that will be machined and therefore cannot be used for locating or clamping. For every surface selected for locating the program have to determine if it is suitable by checking first the selected surface's orientation in relation to the machined surface. This is done by using custom rules of the If-Then-Else variety and SolidWorks' built-in commands through its application programming interface (API). Basically the add-in measures the angle and distance between the two surfaces and, with the help of the rules, decides if the selected surface is suitable. At the moment this means that the angle between the two surfaces have to be between $90^{\circ}$ and $270^{\circ}$.

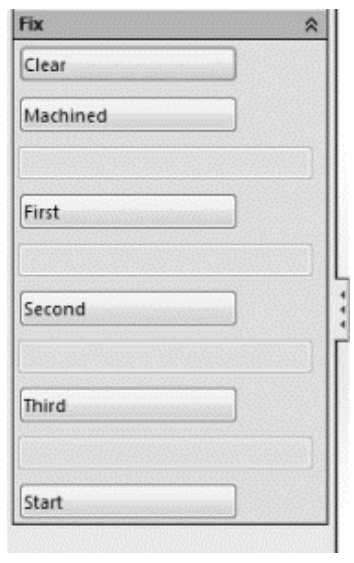

Fig. 4. Property Manager Page.

After successful selection of the to-be-machined surface(s) the text field under the button shows the number of selected surfaces. After successful selection of a locating surface the text field under the button shows "OK". The clamping surfaces are selected by the program and there is 
no indications for selection in the Property Manager Page.

\section{DETERMining THE LOCATING ELEMENTS' POSITIONS.}

As it was stated before, with the location of parts it is crucial the locating elements to be correctly positioned. Those positions directly depend on the surface's shape (rectangle, circle, polygon), dimensions and if it's solid or not are there holes, steps etc. (Fig.5.)).
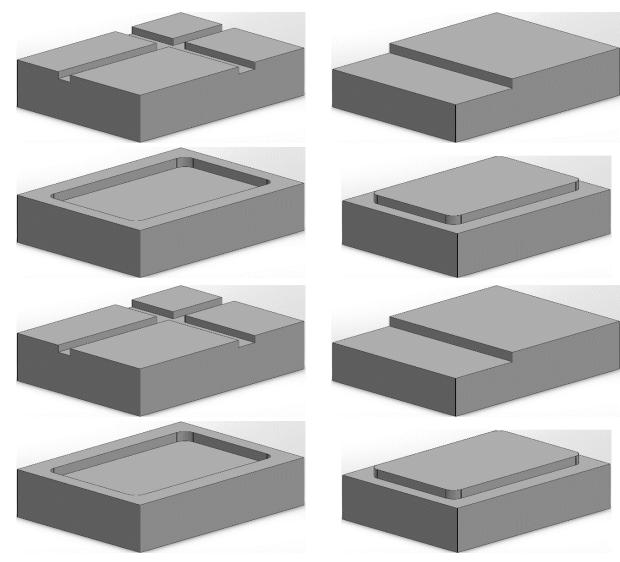

Fig. 5. Surfaces with different features.

When the surface has simple shape (solid square or circle) the positions are easily determent but with complicated shapes and/or additional features the task becomes more and more complicated. The program needs to "understand" what portion of the part it can use for location and that's achieved whit the help of the user and set of rules, which allow the program to find other surfaces in the same plane without the user selecting all of them.

The rules are using SolidWorks' option, available through the application programming interface (API). After the user selects a surface and presses a button, the program determines its orientation by extracting its normal vector data and gets the coordinates of the end points. Then the program checks every surface of the part for others with the same orientation and in the same plane. If there are such surfaces their end points' coordinates are added to those of the selected surface. Example for the used rules:

Automated selection of a surface. Extraction of the normal vector's data.

IF the vector data match the vector data of the surface selected by the user THEN;

IF the surface lies in the same plane as the surface selected by the user THEN;

Collecting the coordinates of the surface's end points.

\section{ELSE}

\section{ELSE}

Automated selection of the next surface;

Automated selection of the next surface.

Those and other rules of the same type are included into cycles, which are repeated until every surface is compared to the selected surface.

After the comparison is concluded, a new sketch is created on the selected surface. The coordinates gathered from all end points are transformed to the local coordinate system of the sketch and the maximal and minimal values for each axis are determined $-\mathrm{x}_{(1 \mathrm{max})}, \mathrm{x}_{(1 \mathrm{~min})}, \mathrm{y}_{(1 \mathrm{max})}, \mathrm{y}_{(1 \mathrm{~min})}$. Those values are used for the calculation of a center point :

$X 10=\frac{X \max +X \min }{2}, Y 10=\frac{Y \max +Y \min }{2}$

The locating points are going to be calculated in a relation to this center point in such manner, that they will imitate the hole pattern on the base plate. Next the distance between the minimal and maximal value for each axis is calculated:

$$
\begin{gathered}
\Delta X=X_{1 \max }-X_{1 \min }, \Delta Y=Y_{1 \max }-Y_{1 \min } \\
\mathrm{kx}_{\mathrm{x}}=\left[\frac{\Delta \mathrm{x}}{0,050}\right], \mathrm{ky}=\left[\frac{\Delta \mathrm{y}}{0,050}\right]
\end{gathered}
$$

The coefficient shows the multiplicity of the distance to the step of the holes on the base plate $(50 \mathrm{~mm})$ rounded down to an integer ( means the floor of , i.e. the largest integer less than or equal to ). Those coefficients are used for calculation of the coordinates for the location elements so the round down guaranties that they will be between the endpoints of the surface. The coordinates are calculated as follows:

$$
\begin{aligned}
& u_{13}=u_{10}+k_{u} * 0,025 ; \\
& v_{11}=v_{10}-k_{v}^{*} 0,025 ; \\
& v_{12}=v_{10}+k_{v}^{*} 0,025 ; \\
& v_{13}=v_{11}+* 0,050
\end{aligned}
$$

The values for are taken from equations (2) and (3) depending on which value of is greater - for and for . The same logic is applied for the values of . In equations (4) is multiplied by half the step of the holes on the base plate, so the symmetry of the locating points according to is consistent to the symmetry of the holes. On the base plate there are no pin-holes on the axes of the plate that's why in the equation for there is additional round down without it in the cases when is odd the coordinate comes between the holes.

With the calculated coordinates the program creates points in the sketch and closes it. Next there are checks on whether the points lie on the surface (or surfaces). The task of those checks is to avoid placing a locating element in a hole, grove or outside the surface. If at least one point is not on the surface (or surfaces) (Fig.6.a) the program implements one or series of corrections aiming to get all points on the surface.

The corrections can be:

- mirror reversal of the points to one axis (Fig.6b);

- single or multiple displacement of all points along one or both axes at distance equal to portion of the free space (Fig.6c), i.e. from (2) and (3):

(6)

- recalculation of the coordinates with coefficient, with the possibility of repetition until ;

- $\quad$ swapping the coefficient (Fig.6d).

The different corrections can be combined in various 
ways so after one type of correction to be executed multiple corrections of different type. For example: after recalculation of the coordinates with coefficient to be executed multiple displacements until reaching, after which another recalculation with to be implemented end so on until. Of course any subsequent correction is executed if at least one point doesn't lie on the surfaces. a)
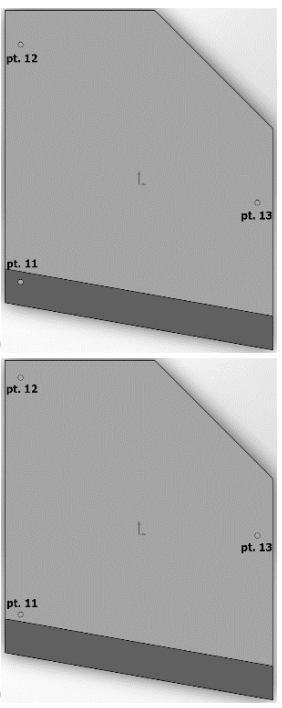

b)

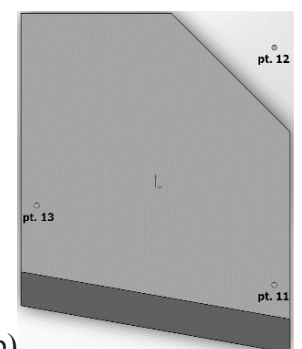

d)

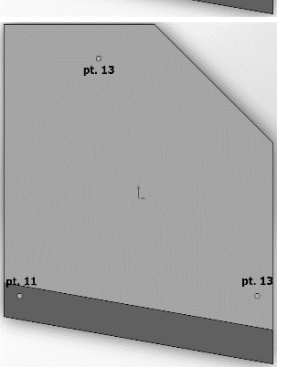

Fig. 6. Locating points - a) calculated coordinates; b), c), d) with corrections.

When all possible corrections are implemented and at least one point doesn't lie on the surfaces the program shows message to the user, stating that the selected surface is not suitable for this type of locating.

The checks and corrections are the same for solid surfaces and surfaces with different features. The program finds the features without the help of the user, however if the user want to use a tiered surface for location he needs to select one surface for each plane. A tiered surface is considered when two or more surfaces have same orientation but lie on different parallel planes. The reason why the user has to select one surface for each plane is to avoid placing locating element on the bottom of any feature (e.g. on the bottom of a blind hole).

When $\left[\frac{0,5 *(v 12-v 11)}{0,050}\right]$ re lying on the surfaces, the program checks if the center of gravity (its projection on the plane of the surface) lies in the triangle of the three points. If it does, then the determination of coordinates is completed successfully, otherwise additional corrections are implemented. The corrections consist of displacement of one or two points along one or both axes at distance multiple of $50 \mathrm{~mm}$ (Fig.7.). After each correction all the points are checked whether they still lie on the surface. If there is no way the center of gravity to lie in the triangle the determination of coordinates can't be completed successfully.

After the successful selection of the first surface (i.e. all checks are passed successfully) the user can make selection for second surface. For determining the locating points on the second and third surfaces the center point is projected onto their planes thus ensuring the same symmetry in all planes.
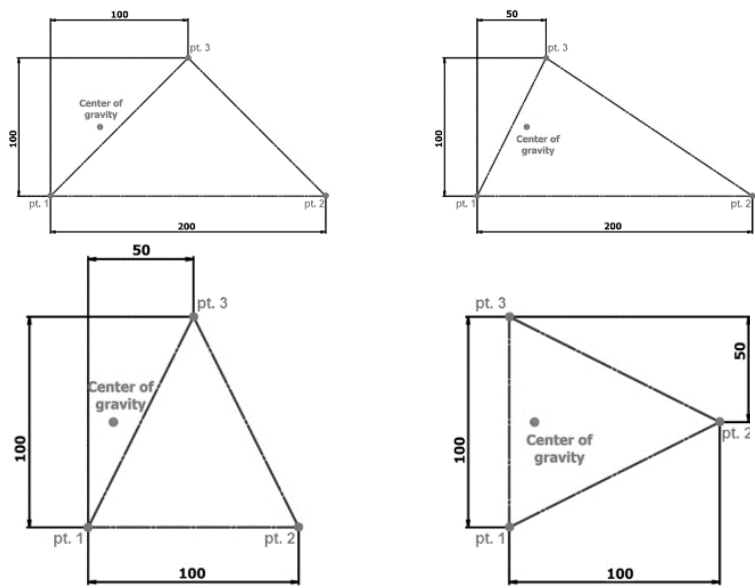

Fig. 7. Positioning the locating points according to the center of gravity.

For the second and third surfaces the coordinates are determined differently than for the first surface. For the second surface the coordinates for one of the axes (the one parallel to the projection of the first surface) are calculated according to :

$$
\begin{aligned}
& \mathrm{u}_{21}=\mathrm{u}_{20}-\left(\left[\frac{\mathrm{u}_{2 \max }-\mathrm{u}_{2 \min }}{0,050}\right] * 0,050\right) / 2 ; \\
& \mathrm{u}_{22}=\mathrm{u}_{20}+\left(\left[\frac{\mathrm{u}_{2 \max }-\mathrm{u}_{2 \min }}{0,050}\right] * 0,050\right) / 2
\end{aligned}
$$

In (6) are the coordinates on the first axis; is the coordinate of the projected point on this axis. The coordinates on the second axis are selected according to the selected adjustable stops (Fig.8.). The coordinates are equal to the height of the adjustable screw (D, C or B) minus the height of the seating pin's head.
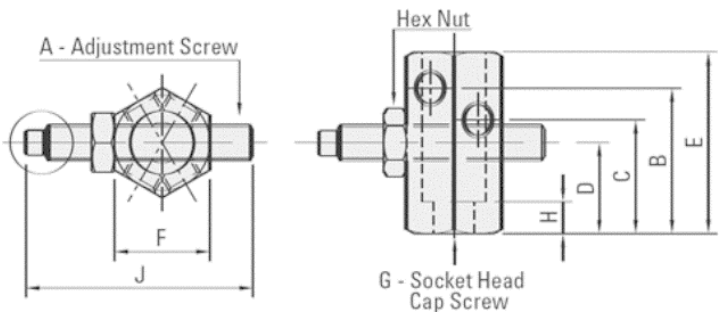

Fig. 8. Adjustable stops. [15]

The height of the adjustable screw is selected so that the center of gravity (its projection on the plane) is between the locating points and the first surface's projection (Fig.9).

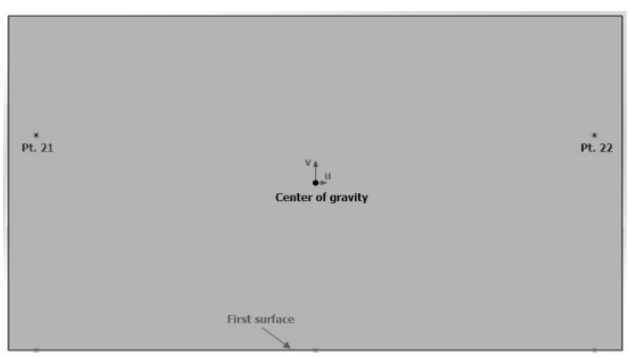

Fig. 9. Position of points 21 and 22 in relation to the first surface and center of mass.

The program creates point with the calculated coordinates and starts checks and corrections, similar to those 
for the first surface. The corrections can be mirror reversal or displacement of one or both points by $50 \mathrm{~mm}$ on each of the axes. If necessary the locating points can be moved below the center of gravity.

For the third surface the coordinate is calculated similar to :

$$
\mathrm{u}_{31}=\mathrm{u}_{30}-\left(\left[\frac{\mathrm{u}_{3 \max }-\mathrm{u}_{3 \min }}{0,050}\right] * 0,050\right) / 2 .
$$

The symbol in (7) is replaced with so that the point of center of gravity is between the locating point and the second surface's projection (Fig.10.). For the second axis the coordinate of the point is selected the same way as . The checks and corrections are same as for the second surface.

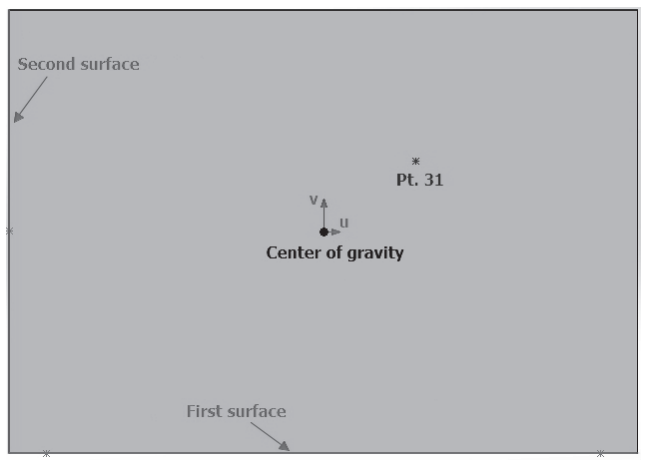

Fig. 10. Position of pt.31 in relation to the first and second surface and the center of mass.

After the successful selection of all three surfaces and all calculations are completed the program transforms all coordinates in global coordinate system and recalculates them so they match the pin-hole pattern on the base plate (Fig.11.). This is done automatically at the end of the user's selection.

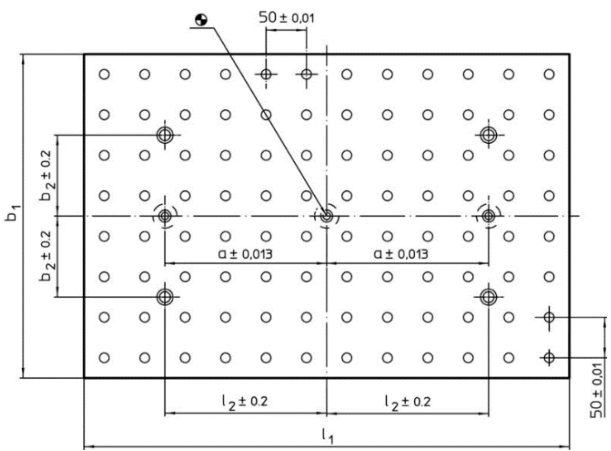

Fig. 11. Base plate [16].

Once the selection process is completed successfully the user can select button "Start" and let the program create a new file of type "ASSEMBLY" (.sldasm). In this file the program places the base plate as fixed part and starts inserting and mating processes for all locating and supporting elements and the part. The program inserts the default configuration of every element and changes it according to the values of coordinates. If it's needed the adjustable stopper's body can be placed on raising elements for additional height. The need, type and dimensions of the raising elements are automatically selected by the system.

All elements are mated together at the calculated positions and the system concludes its work by leaving the new assembly for the user to interact with it (Fig.12.) - to inspect it, to manually correct it if needed, to save the file with selected name and in selected folder etc.

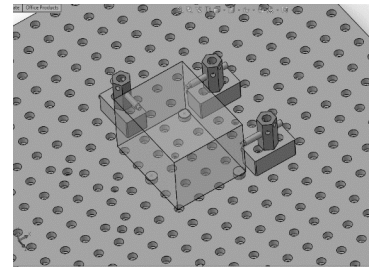

Fig. 12. Assembled fixture with part

\section{Determining the CLAMPING ELEMENTS' POSITIONS.}

The determination of positions of the clamping elements is similar to that of the adjustable stops. It is done in relation to the locating surfaces and elements. The program searches for surfaces that are opposite to the locating surfaces but are not selected as to-be-machined. The clamping points are selected by the same equations with a difference for the side clamps - the positon is calculated not for the middle of the clamp but for the socket that will be outermost from the center of the part (Fig.13.).

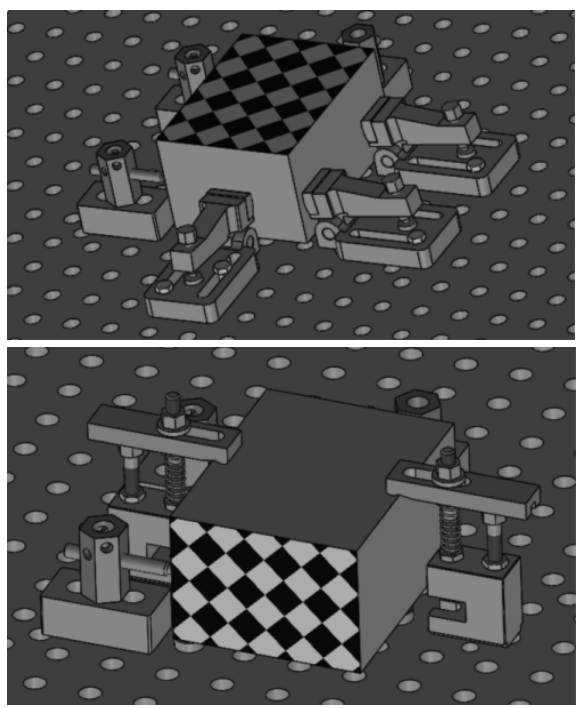

Fig. 13. Clamping (to-be-machined surface in checkered pattern)

\section{CONCLUSIONS}

This paper presents a methodology for determining the positions of the locating and clamping elements in modular fixture design for the 3-2-1 principle. The methodology is consisted of mathematical formulas, rules of the If-Then-Else type and SolidWorks' API functions. The result is optimal positions for the elements according to the workpiece's dimensions and form, the pattern of the base plate's holes and the intended machining surfaces (but not taking the type of machining in consideration). This methodology is included into a system for automated fixture design for prismatic and cylindrical parts. The system is integrated in SolidWorks as an add-in and it uses SolidWorks' API.

This methodology allows accurate automated determination of the positions for the elements. It is 
developed for the 3-2-1 locating principle, and it allows the selection of surfaces with different geometries and containing multiple features (holes, pockets, steps etc.). The input data is selection of surfaces chosen by the user, and the output data is an assembly file consisted of base plate, locating, supporting and clamping elements and the part.

\section{REFERENCES}

[1] F.H. Colvin and L. L. Haas, Jigs and Fixtures: A Reference Book. New York and London: McGraw-Hill Book Company, 1938.

[2] E.K. Henriksen, Jig and Fixture Design Manual. New York, N.Y.: Industrial Press Inc. 1973.

[3] Ts. Kaldachev, P. Hadziski and G.Nikolcheva, "Study wear tool with high-speed milling", Environment. Technology. Resources. 11th International Scientific and Practical Conference, Vol.3, pp.117-121, June 15-17, 2017.

[4] Z.M. Bi and W.J. Zhang, "Flexible fixture design and automation: review, issues and future direction." International Journal of Production Research, Vol.39 (13), pp.2867-2894, 2001.

[5] F. Nixon, Managing to achieve quality. McGraw Hill, Maidenhead, 1971.

[6] Y.(K.) Rong and S. Huang, Advanced computer-aided fixture design. Boston (MA), Elsevier Academic Press, 2005.
[7] Y. Zhu and S. Zang, Modular Fixturing Systems: Theory and Application. Machinery Press, Beijing.

[8] Y. Rong and Y. Zhu, Computer-Aided Fixture Design. Marcel Dekker, Inc., New York, 1999.

[9] G. Nikolcheva and B.Tomova-Raykova, "Modular fixtures stiffness determining", Fourth International Congress "Mechanical Engineering Technologies'04" Varna, Bulgaria, vol.2, pp.102106, September 23-25,2004

[10] W.E. Boyes, Handbook of jig and fixture design, 2nd ed.. Michigan: Society of Manufacturing Engineers, 1999.

[11] I. Boyle, Y. Rong and D.C. Brown, A review and analysis of current computer-aided fixture design approaches. Robotics and Computer-Integrated Manufacturing, Vol. 27 pp.1-12, 2011.

[12] G. Peng, G. Chen, C. Wu, H. Xin and Y. Jiang, Applying RBR and CBR to develop a VR based integrated system for machining fixture design. Expert Systems with Applications, Vol. 38, pp. 26-38, 2011

[13] A. Nedyalkov, P. Sabchev and D. Marov, Processing equipment. Tehnika, Sofia, 1987.

[14] G. Nikolcheva, O. Mihaylov, An integrated add-in for locating parts in SolidWorks, Scientific Proceedings Of the Scientific-Technical Union Of Mechanical Engineering, Vol.3, XII International Congress "Machines. Technologies. Materials 2015", Varna, Bulgaria, pp. 15-18, 2015.

[15] Fixtureworks Workholding Technologies. "Products". Available: www.fixtureworks.net. [Accessed: Nov. 17, 2016].

[16] Erwin Halder KG. "Products". Available: www.halder.com. [Accessed: Nov. 17, 2016]. 